\title{
A Computer-Aided-Design Technique for Design of a Six-Section Elliptic Tire-Building Drum*
}

\author{
Keqi FENG $^{* *}$ and Takuzo IWATSUBO**
}

\begin{abstract}
In this investigation, a computer-aided-design method for a six-section elliptic tire-building drum is presented instead of the traditional geometric construction. The mathematical formulation of the proposed CAD technique is discussed in some detail. The efficient recursive equality-constrained quadratic programming method for solving constrained optimization problems is introduced to synthesize the mechanism of the drum. One practical problem is solved to illustrate the method.
\end{abstract}

Key Words: CAD, Mechanism, Optimum, Design, Synthesis of Mechanism, TireBuilding Drum, Recursive Equality- Constrained Quadratic Program. ming

\section{Introduction}

A tire-building drum is a kind of molding device by which green tires are formed in the manufacturing process of tires. The six-section elliptic tire-building drum (refer to Fig. 1), one of the most complicated types, is a kind of planar rigid guiding mechanism consisting of two symmetrical eight-link linkages. From a mechanical theory viewpoint, the major task in designing a tire-building drum is to make dimensional synthesis of a planar multilink mechanism which should simultaneously satisfy several kinds of requirements. Generally, there are two types of methods in practice: (1) the traditional geometric construction, and (2) the computer-aided-design (CAD) method. Though the method of geometric construction is relatively simple, it cannot give every consideration to the requirements because of its limitations. In particular, if interference between links in the mechanism are found when a mold from the

* Received 18th November, 1988. Paper No.88-2030A

* Faculty of Engineering, Kobe University, Rokko, Nada, Kobe, 657, Japan solution of geometric construction is kinematically examined, the same procedure of geometric construction must be repeated. The optimization technique, as introduced by ${ }^{(1)(2)}$, permits quick and reasonably accurate synthesis of the mechanism, provided it is fairly simple. However, the increasing complexity of new types of tire-building drums and need for their kinematic imitation on a personal computer screen require a more powerful $\mathrm{CAD}$ technique for the design of tire-building drums. In this investigation, a new CAD method for the six-section elliptic tire-building drum is presented. Algorithms and software which can be used on a personal computer are developed. Three features of the method presented in this paper are as follows: (1) suitable combination of tile arrangement and point determination (two steps of the design procedure); (2) the efficient recursive equality-constrained quadratic programming method is introduced to determine points; and ( 3 ) a computer display and drawing system are used to imitate the practical working situation of tire-building drums so that direct visual evaluation of the solution is possible.

The fundamental requirements for tire-building 


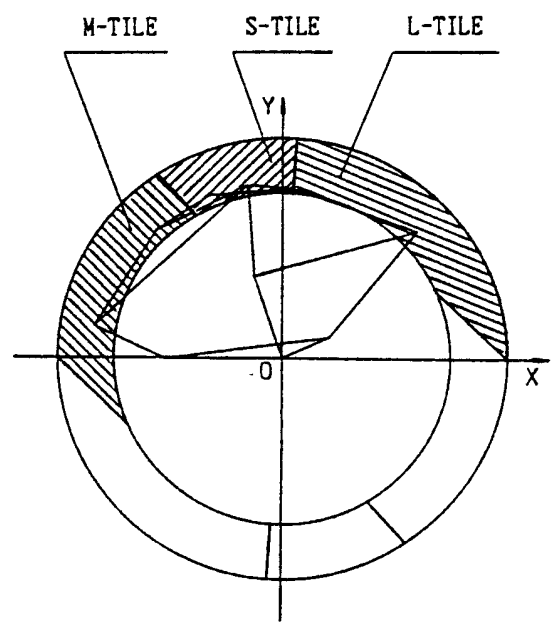

EXPANDED STATE

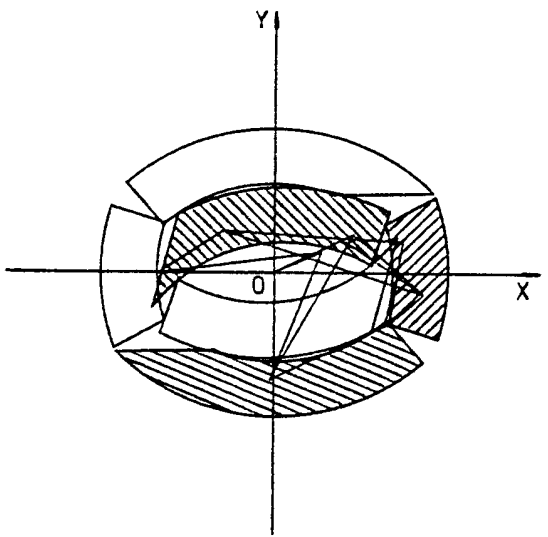

COLLAPSED STATE

Fig. 1 Two states of the drum

drum design, such as the ability to remove tires, the working stability, the folding ability, and the construction practicability, are related to and restricted by each other. To deal with them, the whole procedure of drum design is divided into the following steps :

(1) Tile arrangement;

(2) Tile display;

(3) Point determination (mechanism synthesis)

(4) Checking suitability;

(5) Computer drawing.

The following sections of this paper will focus on the main aspects of the mathematical formulation of tile arrangement and point determination.

\section{Mathematical Formulation}

\section{1 Arrangement tiles (refer to Fig. 2)}

Given parameters:
$D=2 R$
The outside diameter of the drum.
$D_{1}=2 R_{1}$
The inside diameter of the drum.

Selective parameters:

$L^{\prime}$ : The outside diameter of the drum in the collapsed state; The condition $L^{\prime}<L=\pi D_{1}$ should be satisfied ( $L$ is the inside perimeter of the formed tire.).

$\theta:$ The angle corresponding to the longer arc of the approximate ellipse; this influences the shape of the drum in the collapsed state.

$R_{f}:$ The distance between point $F$ and the center of either $L$-tile or $S$-tile; this can be determined according to the requirement of construction reasonableness of the drum.

$\theta_{i}:$ The space angle; $\theta_{i}>0$.

$\theta_{j}$ : The correction angle; this influences the position of $M$-tile and point $E_{2}$, and can be adjusted automatically by the program.

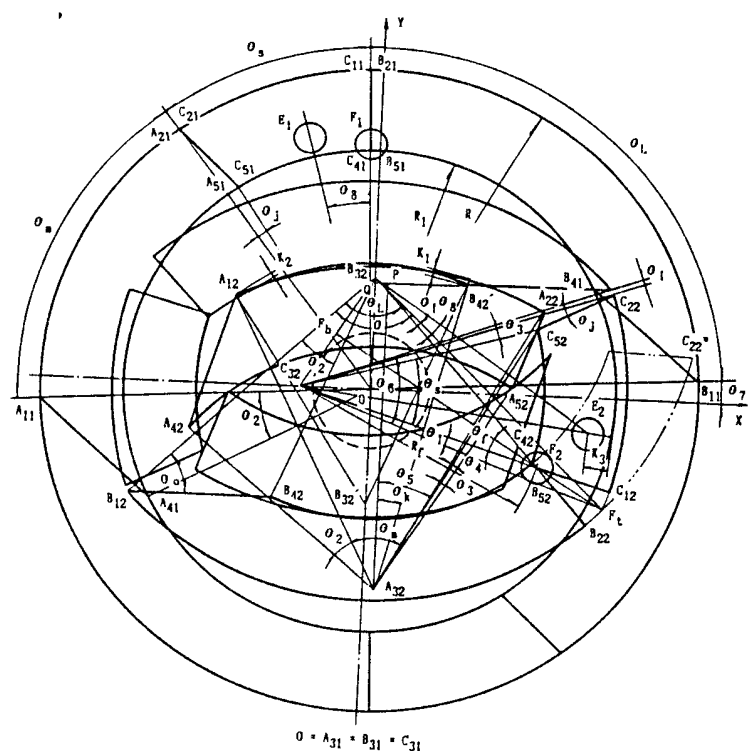

Fig. 2 Arranging tiles of the drum

$\theta_{k}:$ The adjustable angle; this influences the central position of $M$-tile and can be adjusted automatically by the program.

$K_{1}$ : The allowance of the embedded component of $M$-tile at point $B_{24}$.

$K_{2}$ : The allowance of the embedded component of $M$-tile at point $A_{12} ; K_{1}$ and $K_{2}$ influence the longitudinal position of $M$-tile.

$K_{3}$ : The minimum allowable distance between point $E_{2}$ and the outside arc of $S$-tile.

$\theta_{0}:$ The interference angle of $L$-tile and $M$-tile.

$\theta_{7}$ : The position angle; this angle influences the positions of $L$-tile, $M$-tile, and $S$-tile of the drum in the expanded state.

$\Delta \theta_{j}:$ The adjustment step of $\theta_{j}$. 
$\Delta \theta_{k}:$ The adjustment step of $\theta_{k}$.

When the drum changes from the expanded state to the collapsed state, as showed in Fig. 2, its external shape changes from a circle to an approximate ellipse which constitutes four arcs of equal radius $(R)$. The shorter ratio of the perimeter of the drum from the expanded state to the collapsed state is

$i=L^{\prime} / \pi D$.

The sum of central angles corresponding to the four arcs of the approximate ellipse is $2 \pi i$. If $\theta$ is given, the angle corresponding to the shorter arc of the perimeter of the approximate ellipse can be obtained thus:

$\theta_{6}=\pi i-\theta$.

Both the longer and shorter radius of the approximate ellipse can be calculated as follows:

$A=D\left(2 \sin ^{2} \theta_{6} / 4+\sin \theta / 2\right)$,

$B=D\left(2 \sin ^{2} \theta / 4+\sin \theta_{6} / 2\right)$.

$L$-tile is arranged such that its outside arc coincides with the longer arc of the approximate ellipse. The coordinates of the center of $L$-tile are then

$B_{32 x}=0, B_{32 y}=(D-B) / 2$.

$S$-tile is arranged such that its outside arc coincides with the shorter arc of the approximate ellipse. The coordinate of the center of $S$-tile are then

$C_{32 x}=-(D-A) / 2, C_{32 y}=0$.

Since the centers of $L$-tile and $S$-tile coincide in the expanded state, point $F$ on both $L$-tile and $S$-tile in the collapsed state must be located in the middle of a vertical line $F_{b} F_{t}$ between point $B_{32}$ and point $C_{32}$. When $R_{f}$ is specified, the position of point $F$ in the collapsed state can be determined as follows:

$$
\begin{aligned}
& F_{2} F_{b}=\sqrt{R_{f}^{2}-\left(B_{32} C_{32} / 2\right)^{2}}, \\
& F_{t} F_{b}=\sqrt{R^{2}-\left(B_{32} C_{32} / 2\right)^{2}}, \\
& \theta_{f}=\arcsin \left(B_{32} C_{32} / 2 R_{f}\right), \\
& F_{t} F_{2}=F_{t} F_{b}-F_{2} F_{b} .
\end{aligned}
$$

Because of the coincidence of point $C_{12}$ with point $B_{22}$ in the expanded state, the following results can be obtained:

$$
\begin{aligned}
& C_{12} F_{2}=B_{22} F_{2}=R-R_{f}, \\
& C_{12} F_{t} \\
& \quad=\sqrt{C_{12} F_{2}^{2}+F_{t} F_{2}^{2}-2 C_{12} F_{2} F_{t} F_{2} \cos \theta_{f}}, \\
& \theta_{1}=\arcsin C_{12} F_{t} / R .
\end{aligned}
$$

The central angle of $L$-tile is

$$
\theta_{L}=\theta-\theta_{1} \text {. }
$$

The coordinates of all points of $L$-tile can be determined as follows:

$$
\begin{aligned}
& \theta_{2}=\arcsin \left(D / D_{1} \sin \theta_{0}\right)-\theta_{0}, \\
& B_{42 x}=-R_{1} \sin \left(\theta / 2-\theta_{2}\right), \\
& B_{42 y}=B_{32 y}-R_{1} \cos \left(\theta / 2-\theta_{2}\right), \\
& B_{12 x}=-R \sin \theta / 2, \\
& B_{12 y}=B_{32 y}-R \cos \theta / 2,
\end{aligned}
$$

$$
\begin{aligned}
& B_{22 x}=R \sin \left(\theta / 2-\theta_{1}\right), \\
& B_{22 y}=B_{32 y}-R \cos \left(\theta / 2-\theta_{1}\right), \\
& F_{2 x}=R_{f} \sin \left(\theta / 2-\theta_{1}\right), \\
& F_{2 y}=B_{32 y}-R_{f} \cos \left(\theta / 2-\theta_{1}\right) .
\end{aligned}
$$

The central angle of $S$-tile is

$$
\theta_{s}=\theta_{6}-\theta_{1}-\theta_{i} \text {. }
$$

The coordinates of all points of $S$-tile can be determined:

$$
\begin{aligned}
& C_{12 x}=C_{32 x}+R \cos \left(\theta_{6} / 2-\theta_{1}\right), \\
& C_{12 y}=-R \sin \left(\theta_{6} / 2-\theta_{1}\right), \\
& C_{22 x}=C_{32 x}+R \cos \left(\theta_{6} / 2-\theta_{i}\right), \\
& C_{22 y}=R \sin \left(\theta_{6} / 2-\theta_{i}\right) .
\end{aligned}
$$

In order to efficiently utilize the inner space of the drum in the collapsed state, let $\theta_{j}>0$. Then

$$
\begin{aligned}
& \theta_{3}=\arcsin \left(D / D_{1} \sin \theta_{j}\right)-\theta_{j} \\
& C_{52 x}=C_{32 x}+R_{1} \cos \left(\theta_{6} / 2-\theta_{i}-\theta_{3}\right), \\
& C_{52 y}=R_{1} \sin \left(\theta_{6} / 2-\theta_{i}-\theta_{3}\right)
\end{aligned}
$$

The central angle of $M$-tile is

$$
\theta_{m}=\pi-\theta_{L}-\theta_{s} \text {. }
$$

The position of $M$-tile in the collapsed state can be adjusted within the inner space encircled by $L$-tile and $S$-tile. To use the inner space efficiently, line segment $A_{22} A_{52}$ should be as close to point $C_{52}$ as possible, and the outside arc of $M$-tile should touch the inside $\operatorname{arc}$ of $L$-tile. A small amount of embedding in $L$-tile is permissible. These requirements can be written in compact form as follows:

(1) Let $A_{22} A_{52}$ touch point $C_{52}$,

(2) $A_{32} B_{42^{\prime}} \geqq R-K_{1}$,

(3) $A_{12} B_{32} \leqq R_{1}+K_{2}$.

In this paper, $M$-tile is arranged according to (1) and (2), and point $A_{32}$ is then adjusted by the program so that ( 3 ) can be satisfied. The calculation procedure is given as follows:

given a small value of line segment $Q P$, then $\left.\theta_{h}=\arcsin \left(P B_{42} / R-K_{1}\right)\right)\left(P B_{42^{\prime}}=-B_{42 x}-Q P\right)$.

The coordinates of the center of $M$-tile are as fol. lows:

$$
\begin{aligned}
& A_{32 x}=-B_{42 x}-\left(R-K_{1}\right) \sin \theta_{k}, \\
& A_{32 y}=-B_{42 y}-\left(R-K_{1}\right) \cos \theta_{k} .
\end{aligned}
$$

In the triangle of $A_{22} A_{32} C_{52}$,

$$
\begin{aligned}
& C_{52} A_{32}=\sqrt{\left(C_{52 x}-A_{32 x}\right)^{2}+\left(C_{52 y}-A_{32 y}\right)^{2},} \\
& \theta_{4}=\arcsin \left(R / C_{52} A_{32} \sin \theta_{j}\right)-\theta_{j}, \\
& \theta_{5}=\arctan \left(\left(C_{52 x}-A_{32 x}\right) /\left(C_{52 y}-A_{32 y}\right)\right) .
\end{aligned}
$$

The coordinates of all points of $M$-tile can be determined as follows:

$$
\begin{aligned}
& A_{22 x}=A_{32 x}+R \sin \left(\theta_{5}-\theta_{4}\right), \\
& A_{22 y}=A_{32 y}+R \cos \left(\theta_{5}-\theta_{4}\right), \\
& A_{12 x}=A_{32 x}-R \sin \left(\theta_{m}-\theta_{5}+\theta_{4}\right), \\
& A_{12 y}=A_{32 y}-R \cos \left(\theta_{m}-\theta_{5}+\theta_{4}\right), \\
& A_{12} B_{32^{\prime}}=\sqrt{A_{12 x^{2}}+\left(A_{12 y}+B_{22 y}\right)^{2}} . \\
& \text { If } A_{12} B_{32^{\prime}}>R_{1}+K_{2}, \text { then }
\end{aligned}
$$


let $\theta_{k}=\theta_{k}-\Delta \theta_{k} ; \quad\left(\Delta \theta_{k} \quad\right.$ can be specified arbitrarily)

recalculate the coordinates of the center and other points of $M$-tile; repeat the above procedure until ( 3 ) is satisfied.

Since the centers of $L$-tile and $M$-tile coincide in the expanded state, point $E$ on both $L$-tile and $S$-tile in the collapsed state must be located in the middle of a vertical line between point $B_{32}$ and point $A_{32}$. Suppose that $L$-tile and $S$-tile are connected rigidly, that is, no relative motion between them occurs when the drum changes from expanded state to collapsed state. Point $C_{21}$ becomes point $C_{21}{ }^{*}$. Furthermore, point $A_{21}$ and point $C_{21}$ coincide in the expanded state, and therefore, point $E$ in the collapsed state must be located in the middle of a vertical line between point $A_{22}$ and point $C_{22}{ }^{*}$. Accordingly, when the positions of L-tile and $\mathrm{M}$-tile are determined, point $E_{2}$ can be calculated as follows: the coordinates of point $C_{22}{ }^{*}$ are

$$
\begin{aligned}
& C_{22 x}{ }^{*}=R \sin \left(\theta / 2-\theta_{1}+\theta_{s}\right), \\
& C_{22 y}=B_{32 y}-R \cos \left(\theta / 2-\theta_{1}+\theta_{s}\right) .
\end{aligned}
$$

Suppose that the slopes of $A_{22} C_{22}$ and $B_{32} A_{32}$ are $K 1$ and $K 2$, respectively, and the midpoints of the two line segments are $(X 1, Y 1)$ and $(X 2, Y 2)$, the coordinates of point $E_{2}$ are then

$$
\begin{gathered}
E_{2 x}=\left(K_{1} K_{2}\left(Y_{2}-Y_{1}\right)+K_{1} X_{2}\right. \\
\left.\quad-K_{2} X_{1}\right) /\left(K_{1}-K_{2}\right), \\
E_{2 y}=Y_{1}-\left(E_{2 x}-X_{1}\right) / K_{1} .
\end{gathered}
$$

To meet drum construction requirements, the following inequality must be satisfied :

$$
R-C_{32} E_{2}>K_{3} \quad\left(C_{32} E_{2}=\sqrt{E_{2 y}^{2}+\left(E_{2 x}-C_{32 x}\right)^{2}}\right)
$$

If not, let $\theta_{j}=\theta_{j}-\Delta \theta_{j} ; \quad\left(\Delta \theta_{j}\right.$ can be specified arbitrarily)

rearrange $M$-tile and repeat the above procedure until it is satisfied.

Up to this point, the positions of the three tiles in the collapsed state have been determined and calcula. tions of the coordinates of all relevant points have been performed. The coordinates of the points in the expanded state can easily be calculated when the position angle $\theta_{7}$ is specified (refer to Fig. 2).

The displacement matrices of the three tiles from the expanded state to the collapsed state can be calculated as follows ${ }^{(3)}$ :

$$
\begin{aligned}
{\left[D_{e c}\right]_{i} } & =\left[\begin{array}{ccc}
M_{12 x} & M_{22 x} & M_{32 x} \\
M_{12 y} & M_{22 y} & M_{32 y} \\
1 & 1 & 1
\end{array}\right]\left[\begin{array}{ccc}
M_{11 x} & M_{21 x} & M_{31 x} \\
M_{11 y} & M_{21 y} & M_{31 y} \\
1 & 1 & 1
\end{array}\right]^{-1}, \\
i & =L, m, s, \\
M & =A, B, C .
\end{aligned}
$$

\subsection{Synthesis of the mechanism (Point determi- nation)}

For the convenience of the following discussion, the coordinate system is based on link 8 with point 0 as the origin.

The problem of point arrangement is now changed to the optimal synthesis of the 8-link mechanism with one degree of freedom (refer to Fig. 3).

\section{(1) Design Variables}

$$
X=\left(X_{1}, X_{2}, \cdots \cdots, X_{14}\right)^{T} \text {. }
$$

The coordinates of points $F$ and $E$ have been calculated during tile arrangement and are not, therefore, included in the design variables.

(2) Inequality Constraints

It is more convenient in practice that the construction requirements of the drum are given in the polar coordinate system. Transformations between the Cartesian coordinate system and the polar coordinate system are necessary. The relationships between them are indicated below.

$$
\left(X_{2 i-1}, X_{2 i}\right) \cdots\left(l_{i}, \phi_{i}\right) \quad i=1,2, \cdots, 7 \text {. }
$$

Inequality constraints can be written in the following compact from, that is :

$$
\begin{aligned}
& g_{2 n-1}(X)=l_{n \min }-l_{n} \leqq 0, \\
& g_{2 n}(X)=l_{n}-l_{n \max } \leqq 0, \\
& g_{2 n+13}(X)=\psi_{n \min }-\psi_{n} \leqq 0, \\
& g_{2 n+14}(X)=\psi_{n}-\psi_{n \max } \leqq 0 . \\
& n=1,2, \cdots, 7 \\
& (3) \quad \text { Equality Constraints } \\
& h_{1}(X)=\left[\left(D_{2}-B_{1}\right)^{T}\left(D_{2}-B_{1}\right)\right. \\
& \left.-\left(D_{1}-B_{1}\right)^{T}\left(D_{1}-B_{1}\right)\right]^{2}=0 \\
& h_{2}(X)=\left[\left(A_{2}-O_{1}\right)^{T}\left(A_{2}-O_{1}\right)\right. \\
& \left.-\left(A_{1}-O_{1}\right)^{T}\left(A_{1}-O_{1}\right)\right]^{2}=0 \\
& h_{3}(X)=\left[\left(G_{2}-C_{1}\right)^{T}\left(G_{2}-C_{1}\right)\right. \\
& \left.-\left(G_{1}-C_{1}\right)^{T}\left(G_{1}-C_{1}\right)\right]^{2}=0 \\
& h_{4}(X)=\left[\left(I_{2}-H_{2}\right)^{T}\left(I_{2}-H_{2}\right)\right. \\
& \left.-\left(I_{1}-H_{1}\right)^{T}\left(I_{1}-H_{1}\right)\right]^{2}=0
\end{aligned}
$$

where

$$
\begin{aligned}
& D_{2}=\left[D_{e c}\right]_{m} D_{1} \\
& A_{2}=\left[D_{e c}\right]_{L} A_{1} \\
& G_{2}=\left[D_{e c}\right]_{L} G_{1}
\end{aligned}
$$

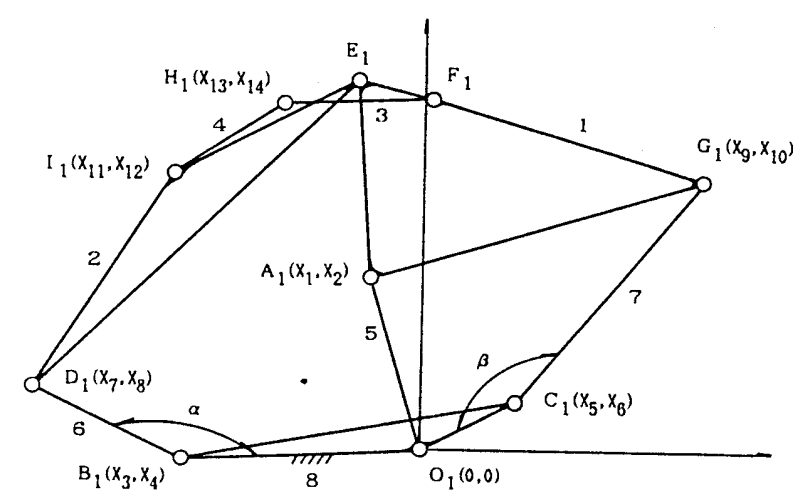

Fig. 3 Determining points of the drum 


$$
\begin{aligned}
& I_{2}=\left[D_{e c}\right]_{m} I_{1} \\
& H_{2}=\left[D_{e c}\right]_{s} H_{1}
\end{aligned}
$$

(4) Objective Function

The transmission angles of the instability movement are

$$
\begin{aligned}
& \alpha=\arccos \left(D_{1} B_{1}^{2}+l_{2}^{2}-l_{4}^{2}\right) /\left(2 D_{1} B_{1} l_{2}\right), \\
& \beta=\arccos \left(G_{1} C_{1}^{2}+l_{3}^{2}-l_{5}^{2}\right) /\left(2 G_{1} C_{1} l_{3}\right) . \\
& \text { The objective function is } \\
& F(X)=W_{1}\left(\alpha-V_{1}\right)^{2}+W_{2}\left(\beta-V_{2}\right)^{2},
\end{aligned}
$$

where $W_{1}$ and $W_{2}$ are two weighted factors. $V_{1}$ and $V_{2}$, two control factors which can control the transmission angles $\alpha$ and $\beta$ in the optimization process, are applied in the objective function as a trade-off between the working stability and the folding ability of the drum. Detailed discussions may be referred to in $^{(1)}$.

Based on the above discussions, the problem of dimensional synthesis of the eight-link mechanism of the drum now becomes a nonlinear mathematical programming problem defined as :

$$
\begin{array}{lll}
\text { minimize } & F(X), \quad X \in R^{14} \\
\text { subject to } & h_{u}(X)=0, \quad u=1,2,3,4 \\
& g_{v}(X) \leqq 0, \quad v=1,2, \cdots, 28
\end{array}
$$

\section{Optimization Method}

Nonlinear programming, by which almost all engineering optimization problems can be construct. ed, is the most difficult of optimization problems. Indeed there is no general agreement on the best approach, especially in different applications. In the early stage of this investigation, the sequential unconstrained minimization method (SUMT) was adopted. Since both equality and inequality constraints are involved in (42), the penalty function is formed as :

$$
\begin{aligned}
& P(X, r)=F(X)-r \sum_{v=1}^{28} 1 / g_{v}(X) \\
& \quad+(1 / \sqrt{r}) \sum_{u=1}^{4}\left[h_{u}(X)\right]^{2}
\end{aligned}
$$

where $r$ is a penalty factor.

This method suffers from some computational disadvantages and is not entirely efficient despite the simplicity of the method and ease of software development.

Another approach in solving (42) is to identify the active inequality constraints at any stage of a solution and consider these as added equality constraints. The problem then takes the from

$$
\begin{array}{ll}
\text { minimize } & F(X), \quad X \in R^{14} ; k \\
\text { subject to } & C_{i}(X)=0, \quad i \in I_{a},
\end{array}
$$

where $\mathbb{I}_{a}^{k}$ denotes the active constraints at the $k$ th stage of the optimization.

The recursive equality-constrained quadratic programming (REQP) method considers the following subproblem: minimize $g^{T} d+0.5 d^{T} H^{k} d, d \in R^{14}$;

$$
\text { subject to } C^{k}+A^{k} d-0.5 r^{k} \lambda^{k}=0 \text {, }
$$

where $g=\left[\partial F / \partial X_{1}, \partial F / \partial X_{2}, \cdots, \partial F / \partial X_{14}\right]^{T}$.

$H^{k}$ represents the Hessian matrix or its approximation based on the Lagrangian

$$
L=F(X)+\lambda^{T} C^{k} \text {. }
$$

with $C^{k}$ indicating the vector of current values for the $m$ active constraints. The rows of the matrix $A^{k}$ contain the gradients of the active constraints such that

$$
\begin{aligned}
A & =\left[a_{1}, a_{2}, \cdots, a_{m}\right] \\
& =\left[\begin{array}{ccc}
\partial C_{1} / \partial X_{1} & \partial C_{1} / \partial X_{2} \cdots \partial C_{1} / \partial X_{14} \\
\vdots & \vdots & \vdots \\
\partial C_{m} / \partial X_{1} & \partial C_{m} / \partial X_{2} \cdots \partial C_{m} / \partial X_{14}
\end{array}\right]
\end{aligned}
$$

$r^{k}$ is the current value of the penalty parameter. The closed form solution for the EQP subproblem is

$$
d=-\left(H^{k}\right)^{-1}\left[\left(A^{k}\right)^{T} \lambda^{k}+g^{k}\right]
$$

with the Lagrange multiplier estimate

$$
\begin{gathered}
\lambda^{k}=\left[0.5 r^{k} I+A^{k}\left(H^{k}\right)^{-1}\left(A^{k}\right)^{T}\right]^{-1} \\
{\left[-A^{k}\left(H^{k}\right)^{-1} g^{k}+C^{k}\right],}
\end{gathered}
$$

where $I$ is the identity matrix. The merit function, which is used in a line search in $\eta$ such that $X^{k+1}=X^{k}$ $+\eta d$, is

$$
P\left(X, r^{k}\right)=F(X)+\left(1 / r^{k}\right) C^{T} C .
$$

Reference ${ }^{(6)}$ has proven that this algorithm pos-

Table 1 The coordinates of hinge points of the drum

\begin{tabular}{|c|c|c|c|c|}
\hline \multirow{2}{*}{ POINT } & \multicolumn{2}{|c|}{ EXPANDED STATE } & \multicolumn{2}{c|}{ COLL.APSED STATE } \\
\cline { 2 - 5 } & \multicolumn{1}{|c|}{$X$} & \multicolumn{1}{c|}{$Y$} & \multicolumn{1}{c|}{$X$} & \multicolumn{1}{c|}{$Y$} \\
\hline A & -33.333 & 100.273 & 94.473 & 46.609 \\
B & -144.053 & -5.317 & -144.053 & -5.317 \\
C & 55.435 & 27.771 & 55.435 & 27.771 \\
D & -228.599 & 30.395 & -70.125 & 49.536 \\
E & -41.482 & 212.097 & 179.307 & -26.700 \\
F & 13.247 & 207.578 & 137.413 & -62.204 \\
G & 163.341 & 163.341 & 0.001 & -137.057 \\
H & -87.958 & 199.478 & 153.624 & 37.885 \\
I & -150.579 & 156.764 & 78.205 & 42.165 \\
D & 0.0 & 0.0 & 0.0 & 0.0 \\
\hline
\end{tabular}

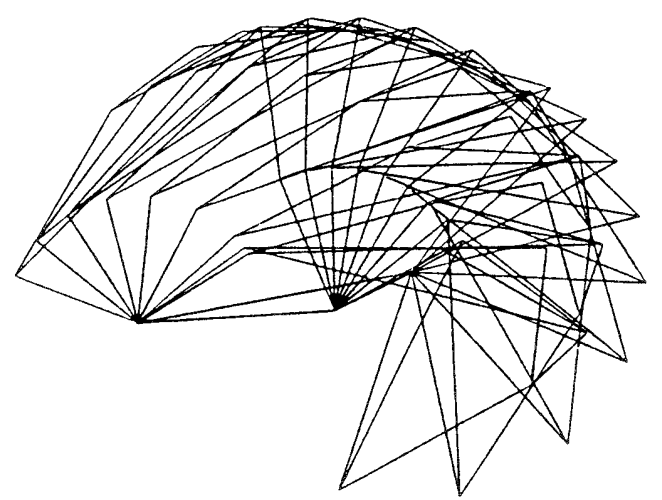

Fig. 4 Kinematic trajectory drawing of the mechanism 


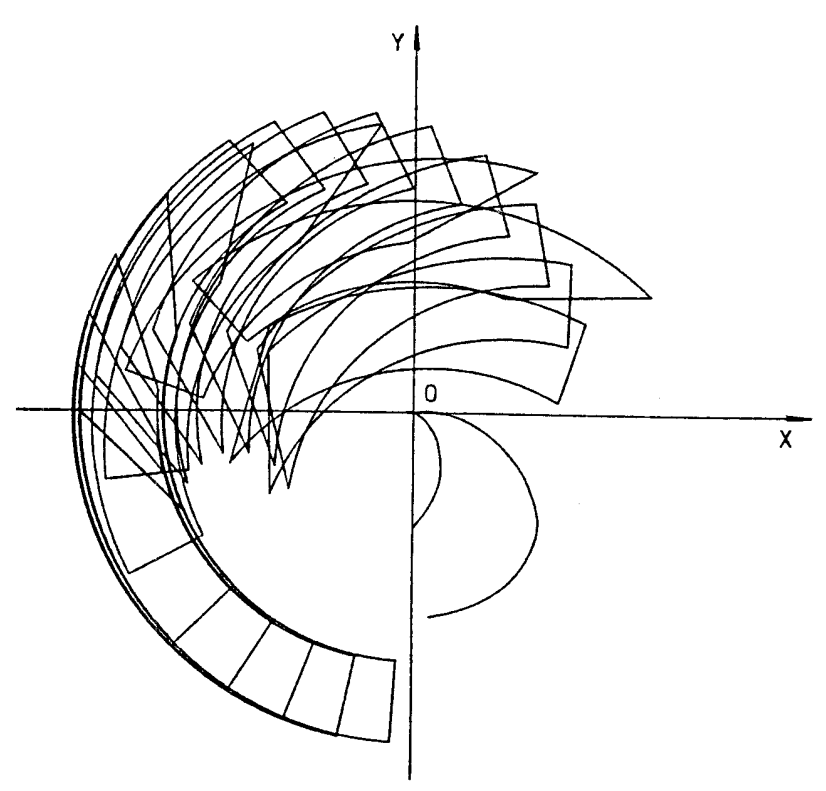

Fig. 5 Interference checking drawing of the drum

sesses global convergence and locally superlinear convergence properties. In order to further increase the efficiency of the REQP method ${ }^{(7)}$, includes the monotonicity analysis technique in the REQP and ${ }^{(5)}$ employs an augmented Laglangian penalty function for both the EQP subproblem and line search. The numerical analysis experience on the design of tirebuilding drums shows that the REQP method is more efficient than the SUMT. Detailed discussions of the REQP method and its improvement can be found in $^{(5) \sim(7)}$.

\section{An Example}

The numerical results of design of one six-section elliptic tire-building drum by the $\mathrm{CAD}$ method presented in this paper are given in Table 1. (Millimeter for length and degree for angle are used below.)

Given parameters : $D=540, D 1=405$; Selectable parameters: $L^{\prime}=1245, \theta=90, R_{f}=208, \theta_{0}=44, \theta_{i}$ $=1, \theta_{j}=9, \theta_{k}=12.8, \theta_{7}=0, K_{1}=3, K_{2}=2, K_{3}=28$

The kinematic trajectory drawing of the mechanism and the interference-checking drawing of $L$-tile and $M$-tile are given in Fig.4 and Fig.5, respectively.

\section{References}

(1) Feng, K. Q. and Zhu, Y.H., Optimum Design of Folding Tire- Building Drums, J. of Tianjin Univ., 3 (1984).

(2) Feng, K.Q. , Stability Analysis of Tire-Building Drums, J. of Tianjin Univ., in press.

(3) Suh, C. W. and Radeliffe, C. W., Kinematics and Mechanisms Design, (1978), John Wiley \& Sons.

(4) Fletcher R., Practical Methods of Optimization, (1980), John Wiley \& Sons.

(5) Chen, C., Kong, W.C., and Cha, J.Z., An EqualityConstrained RQP Algorithm Based on the Aug. mented Lagrangian Penalty Function, ASME paper, Boston, MA, (1987-9).

(6) Biggs, M.C., On the Convergence of Some Constrained Minimization Algorithms Based on Recursive Quadratic Programming, J. Inst. Maths. Applns., 21 (1978).

( 7 Zhou, J. and Mayne, R.W., Monotonicity Analysis and Recursive Quadratic Programming in Constrained Optimization, ASME J. Mech. Tran. \& Auto. in Design, Vol. 107 (1984). 